2

\title{
A new protocol for multiple muscle mapping using nTMS
}

2

(1)

\author{
Fang Jin, Sjoerd Bruijn ${ }^{*}$, Andreas Daffertshofer
}

Faculty of Behavioural and Movement Sciences, Department of Human Movement Sciences, Vrije Universiteit, Institute of Brain and Behavior Amsterdam and Amsterdam Movement Sciences, Van der Boechorststraat 9, 1081 BT Amsterdam, The Netherlands

*Corresponding author: s.m.bruijn@vu.nl

Keywords: nTMS, motor mapping, multiple muscles, cortical representation, active area

\section{Abstract}

Background: Single-pulse transcranial magnetic stimulation is a safe and non-invasive tool for investigating cortical representation of muscles in the primary motor cortex. While nonnavigated TMS has been successfully applied to simultaneously induce motor-evoked potentials (MEPs) in multiple muscles, a more rigorous assessment of the corresponding cortical representation can greatly benefit from navigated transcranial magnetic stimulation (nTMS).

Objective: We designed a protocol to map the entire precentral gyrus using neural navigation while recording responses of eight muscles simultaneously. Here, we evaluated the feasibility, validity, and reliability of this protocol.

Method: $\quad$ Twenty participants underwent conventional (i.e., muscle-based, grid-constrained) and gyrus-based nTMS mapping. For both protocols, we investigated three different stimulation intensities during two consecutive sessions.

Results: $\quad$ The gyrus-based nTMS mapping was received well by all participants and was less time consuming than the grid-constrained standard. On average, MEP amplitudes, latencies, and centre-of-gravity and size of the active areas largely agreed across protocols supporting validity. Intraclass coefficients between sessions unscored the reliability of our protocol.

Conclusion: We designed an nTMS protocol for the simultaneous mapping multiple muscles on the cortex. The protocol takes only about ten minutes per participant when including as many as eight muscles. Our assessments revealed that the cortical representation of multiple muscles can be determined with high validity and reliability. 


\section{Introduction}

Single-pulse transcranial magnetic stimulation (TMS) is a non-invasive and painless technique that allows for monitoring neurophysiological alterations of the human motor cortex [1, 2]. When a TMS coil discharges at a suitable intensity, the time-varying magnetic field will induce transient currents causing depolarisation of axons of nerve cells [3]. This will elicit a motor-evoked potential (MEP), which can be recorded in contralateral target muscles. Amplitudes and latencies of these MEPs can reveal the excitability and conduction times of the cortical-spinal tract. Both have been conceived as valid parameters of TMS motor mapping [4]. Neuroscientists and physicians alike utilised TMS motor mapping to evaluate motor cortical plasticity [5], to plan brain tumour surgery [6] and to follow the recovery after stroke [7]. There is ample evidence that the maximum MEP elicited using TMS is closer to the site found using direct cortical stimulation - the gold standard in motor mapping - than that found using magnetoencephalography (MEG) [8] and functional magnetic resonance imaging (fMRI) [9].

In traditional TMS motor mapping procedures, a grid is printed on a cap worn by the subject. This grid serves to manoeuvre the coil and stimulate at the adequate position [10, 11]. Navigated TMS (nTMS) has been developed to substitute the cap, yet the grid-based positions remain. The grid can be displayed on an anatomical scan (typically an MRI) allowing to position the coil according to neuroanatomy. This reduces the error from relative movements of cap and head [12]. That advance has been improved by also accounting for the orientation of the coil, next to the mere positioning [13]. The most recent addition in nTMS systems has been positioning with instantly assessed electromyography (EMG), to display MEP parameters (almost) online on the stimulated sites of the cortex. By this, one can, e.g., a create map of MEP amplitudes for a certain muscle during an experiment (rather than during post-processing) [14].

These advances in technology have led to improvements in experimental protocols. As said, for TMS without neural navigation, a grid on a cap serves to guide coil positioning [10]. In nTMS, the grid can be readily repositioned around the so-called hotspot of a target muscle, i.e. the centre of the area of interest for subsequent assessments, e.g., with different stimulation intensities $[15,16]$ quantified relative to the muscle-specific resting motor threshold (RMT) [17]. Littmann and co-workers [18] designed an optimal grid by increasing the number of points over the precentral gyrus and reducing the number of points far away from it. Despite these improvements, however, it remains a challenge to move the coil between closely neighbouring points. Van De Ruit, Perenboom, Grey [19] compared grid-based procedures to a protocol in which stimulations were applied at pseudorandom positions. Not only did this alternative approach reduce the time needed to map a single muscle, it turned out to be as reliable as the conventional procedures; see also Cavaleri, Schabrun, Chipchase [20]. 
Identifying the cortical map of multiple muscles in one experimental run will be difficult - if at all possible - without neural navigation [10]. Yet, studies exploiting nTMS for that sake are few and far between - see [21] for an exception where up to four muscles were targeted. The focus on single muscle mapping arguably stems from the fact that - in most nTMS protocols - stimulation intensities at a specific percentage of a single muscle's RMT are deemed important. Detecting RMTs for several muscles is laborious because it often involves offline EMG analyses. Quite recently, however, it has been shown that the RMT of a small hand muscle (there, the first dorsal interosseous, FDI) may be similar to the RMT of all upper extremity muscles [16].

All these developments led us to design a new nTMS-based protocol for multiple muscles on the precentral gyrus (primary motor cortex). Here, we illustrate its feasibility, validity, and reliability. As will be shown, our mapping protocol significantly reduces operation times and drastically simplifies procedures. By the same token, it comes with proper validity and reliability. The protocol can provide information about the cortical representations of multiple muscles and the degree to which they overlap. For this, we also submit a new way to define and measure the so-called active area. Through our research, we anticipate changing the nTMS paradigm in research and clinic: from grid- to gyrus-based and from single to multiple muscle mapping.

\section{Materials and methods}

\section{Participants}

Twenty healthy, right-handed volunteers (average age: $29.55 \pm 7.49$, eight females) participated in the study. Prior to the experiment, all participants were screened for contraindications of MRI and TMS through questionnaires [22]. All of them provided signed informed consent. The Edinburgh Handedness Inventory was used to determine hand dominance [23]. The study had been approved by the medical ethics committee of Amsterdam University Medical Center (VUmc, 018.213 NL65023.029.18).

\section{Materials}

Our set-up consisted of three devices: a TMS system, an EMG amplifier, and a neural navigator. Singlepulse TMS was delivered by a Magstim $200^{2}$ stimulator (Magstim Company Ltd., Whitland, Dyfed, UK) using a figure-of-eight coil with $70 \mathrm{~mm}$ windings. Eight bipolar EMG signals were recorded using a 16channel EMG amplifier (Porti, TMSi, Oldenzaal, the Netherlands) and continuously sampled at a rate of $2 \mathrm{kHz}$. The EMG recordings were triggered by the TMS to allow for online EMG-assessments using a custom-made Labview-programme with embedded Matlab functions (designed at our department using Labview 2016, National Instruments, Austin, TX, and Matlab 2018b, The MathWorks, Natick, 
MA). In brief, upon receiving a trigger, peak-to-peak amplitudes and latencies of MEPs were estimated from all EMG signals during the following 500 ms. These parameters, as well as the original EMG signals (duration $=500 \mathrm{~ms}$ ), were sent to the neural navigation software (Neural Navigator, Brain Science Tools, De Bilt, The Netherlands, www.brainsciencetools.com) for online monitoring and storage. The neural navigation software also stored the position and orientation of the coil with respect to the head.

Prior to running the TMS protocol, we acquired the participants' anatomical T1-weighted MRI (3 Tesla Philips Achieva System, Philips, Best, The Netherlands; matrix size $256 \times 256 \times 211$, voxel size $1.0 \times 1.0 \times 1.0 \mathrm{~mm}^{3}$, TR/TE $\left.6.40 / 2.94 \mathrm{~ms}\right)$. The MRI grey matter was segmented using SPM (SPM12, https://www.fil.ion.ucl.ac.uk/spm/software/spm12/).

We considered the first dorsal interosseous (FDI), abductor digiti minimi (ADM), abductor pollicis brevis (APB), flexor pollicis brevis (FPB), extensor digitorum communis (EDC), flexor digitorum superficialis (FDS), extensor carpi radialis (ECR) and flexor carpi radialis (FCR) muscles, which were measured using bipolar electrodes (Blue Sensor N-00-S, Ambu, Ballerup, Denmark), placed after cleaning the skin with alcohol; cf. Figure 1. The ground electrode was attached to the ulnar styloid process. We monitored and kept the electrode impedance below $5 \mathrm{k} \Omega$. During the experiment, the orientation of the TMS coil was held 45 degrees to the sagittal plane, tangential to the scalp. By this, we meant to induce currents in the cortex along the posterior-to-anterior direction. To control the TMS output, we modified the employed Matlab-toolbox (https://github.com/armanabraham/Rapid2) when adjusting intensities and intervals between stimulations.
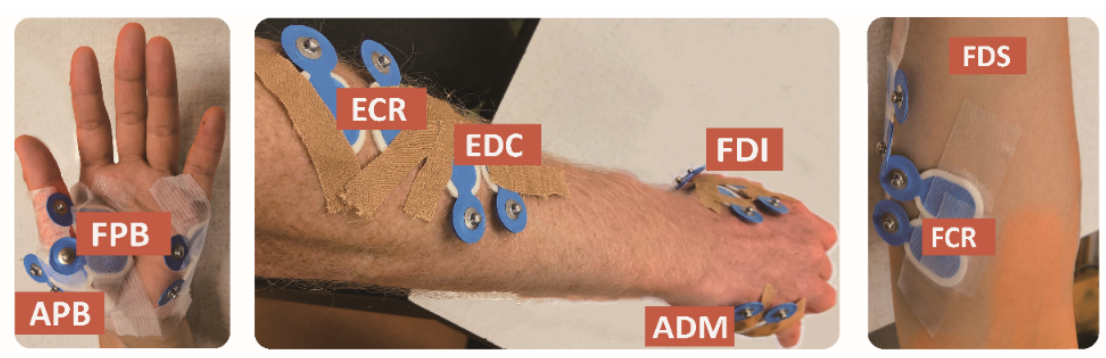

Figure 1. Placement of bipolar electrodes for the first dorsal interosseous (FDI), abductor digiti minimi (ADM), abductor pollicis brevis (APB), flexor pollicis brevis (FPB), extensor digitorum communis (EDC), flexor digitorum superficialis (FDS), extensor carpi radialis (ECR) and flexor carpi radialis (FCR) muscles.

\section{Experimental procedures}

Participants were comfortably seated in an armchair, relaxing muscles of hands and arms. The experiment consisted of two identical sessions, which were separated by one hour to test for test-retest reliability of our outcomes - electrodes were kept fixed to minimise placement errors. The interval of one hour only was set to prevent drying of the conductive electrolyte gel. Each session contained three 
parts: (1) Testing the RMTs for FDI, EDC and FCR; there, we stimulated thirty points near the omegashaped area of the precentral gyrus to identify the respective hotspots defined as the coordinates for which the largest peak-to-peak amplitudes in the corresponding EMG signals could be observed. This served to determine the RMTs for the three target muscles following [24] (i.e. the stimulator output at which peak-to-peak amplitudes were higher than $50 \mu \mathrm{V}$ in five out of ten stimuli). (2) We conducted two sets of stimulations to map the representations of all eight muscles using three intensities each, namely $105 \%$ RMT of FDI, EDC and FCR, respectively. For the first set, we adopted the conventional grid-based method [25] using a square grid $(5 \mathrm{~cm} \times 5 \mathrm{~cm})$ with either the FDI-, EDC- or FCR-hotspot as the centre and applied 80 stimuli. This was immediately followed by a pseudorandom positioning over the whole gyrus ( 40 stimuli), yielding a total of $80+40=120$ stimuli - in the following we consider the total of 120 stimuli for our gyrus-based approach while the grid mapping contained only the first 80 stimuli. (3) We analysed the last 40 stimulations and estimated the hotspots of the other five muscles (ADM, APB, FPB, FDS and ECR) and determined their RMTs.

\section{Offline data processing}

During the measurement, peak-to-peak amplitudes and latencies of the MEPs were estimated. The MEP was defined as the range between the minimum and maximum peak of the EMG signal, and the latency as the onset of the MEP signal (https://github.com/marlow17/surfaceanalysis). The onset was defined as the point in time at which the signal exceeded mean $\pm 1.96 \times$ sd of the signal baseline (100 ms prior to up to the moment of stimulus).

For every muscle and stimulation, we determined whether a MEP was elicited; see Supplementary Material for details. Whenever MEPs were present, the corresponding parameters were included when computing the mean MEP amplitude and latency, the centre-of-gravity of the stimulation surface $\left(\operatorname{CoG}_{x, y, z}\right)$ and the size of the active area, i.e., the size of that surface. We defined the centre-ofgravity as follows [26]:

$$
\operatorname{CoG}_{x}=\frac{\sum M E P_{i} \cdot X_{i}}{\sum M E P_{i}}, C o G_{y}=\frac{\sum M E P_{i} \cdot Y_{i}}{\sum M E P_{i}}, \text { and } \operatorname{CoG}_{z}=\frac{\sum M E P_{i} \cdot Z_{i}}{\sum M E P_{i}}
$$

Here, $M E P_{i}$ represents the peak-to-peak amplitude for stimulation $i$ at position $\left(X_{i}, Y_{i}, Z_{i}\right)$. The definition of the active area is more involved, but we provide more details in the Supplementary Material. All the analyses were performed separately for the $3 \times 2 \times 2$ cases: three different muscle-specific intensities, for grid- and gyrus-based mapping, and for both sessions. The different steps are illustrated in Figure 2. 

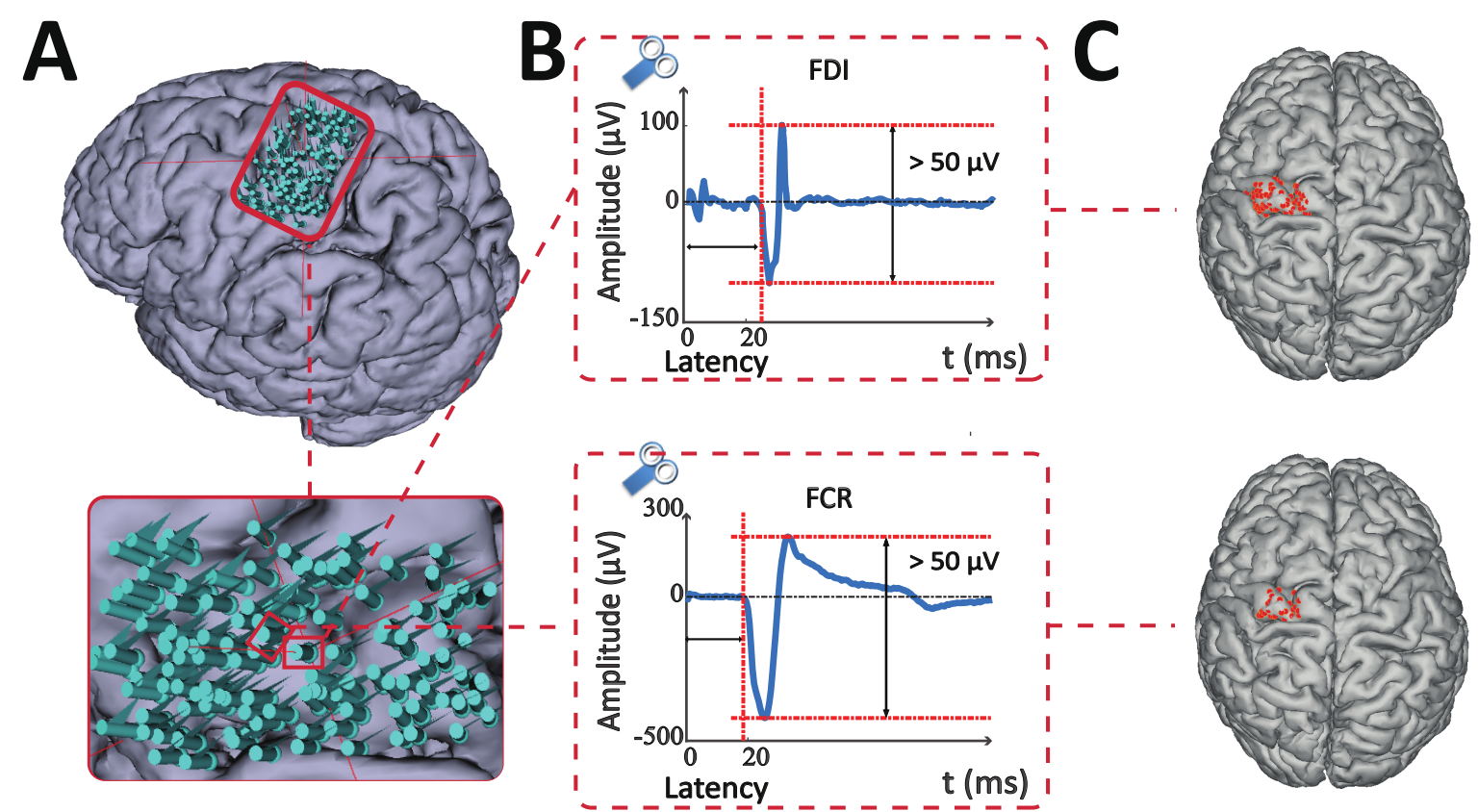

Figure 2. The schematic illustrates the procedures of the experiment and data analysis. (A) The gyrus-based method is illustrated by the points of stimulation projected on subjects' pial MRI surface in the neural navigation system in the above picture. The two points highlighted in the lower panel inlet represent the points of the FDI and FCR representation, respectively, corresponding to the motor evoked potentials (MEPs) shown in column (B): top $=$ FDI, bottom $=$ FCR. The MEPs were defined as the peak-to-peak value of the EMG signal under the provision that it exceeded $50 \mu \mathrm{V}$ and the latency was between 15 to $30 \mathrm{~ms}$. (C) The cortical representations of FDI and FCR, i.e., the muscle-specific points shown in column (A) with proper MEPs.

\section{Statistics}

All statistical analyses were conducted in Matlab. First, we calculated the average values of RMTs over all the subjects and sessions of the eight muscles. Possible differences of RMTs across the eight muscles were assessed using a two-way repeated ANOVA with Muscle and Session as factors, with the consecutive post-hoc assessment after Bonferroni correction. To evaluate the validity of the gyrusbased protocol as compared to the grid-based one, we used Bland-Altman plots analysing the amplitude, latency, $\operatorname{Co} G_{x, y, z}$, and the size of the active area. We did this only for the parameters of the muscles that were targeted with the specific intensity, i.e., we compared the grid-based and the gyrusbased protocol to map the cortical representations of FDI at 105\% RMT of FDI, etc. To test the reliability of our gyrus-based protocol, we used intraclass correlation coefficients (ICC) between Session 1 and Session 2. These were calculated for the MEP amplitude and latency, the $\operatorname{CoG}_{x, y, z}$, and the size of the active area. Effects of stimulation intensity were investigated by estimating the ICCs for all MEP parameters. For the sake of legibility, however, we defined distinct classes of reliability by discretising the ICC-values as follows: Excellent: $0.8 \leq$ ICC; Good: 0.65 IICC < 0.8; Moderate: $0.5 \leq$ ICC < 0.65; Poor: ICC $<0.5$ [20]. We also performed a two-way repeated measures ANOVA with the factors of Intensity and Session on all the parameters (again with Bonferroni correction for multiple comparisons). Prior 
to hypothesis testing, sphericity was verified via Mauchly's test and, if applicable a Greenhouse-

174 All $N=20$ participants completed the experimental procedure without adverse reactions. Overall, of 17520 subjects $\times 8$ muscles $\times 3$ intensities $\times 2$ sessions $=960$ cortical representations, only $1.77 \%$ did not 176 elicit proper MEPs (11 of 320 stimulations in the intensity of 105\% RMT of FDI, 5 of 320 stimulations in the 105\% RMT of EDC and 1 of 320 stimulations in the intensity of 105\% RMT of FCR; see Table S1 in the Supplementary Material for more details). For five subjects we could not detect MEPs for ADM when using the second intensity in both sessions.

Resting motor thresholds

For all the participants and sessions, the average RMTs of the eight muscles were: FDI $(44.90 \pm 1.46 \%)$, $\operatorname{ADM}(47.90 \pm 1.64 \%), A P B(46.15 \pm 1.45 \%), \operatorname{FPB}(46.78 \pm 1.73 \%), \operatorname{EDC}(45.28 \pm 1.50 \%), \operatorname{FDS}(47.75 \pm 1.51 \%)$, ECR (46.55 $\pm 1.50 \%)$ and FCR (48.00 $\pm 1.52 \%)$. The repeated ANOVA revealed a significant effect of Muscle $(F(7,133)=3.63, p=.001)$. There was no significant effect of Session $(F(1,19)=0.85, p=.369)$ or interaction between Muscle and Session $(F(7,133)=1.69, p=.118)$. Post-hoc pairwise comparisons indicated significant differences between EDC-FCR $(p=.020)$ and FDI-FCR $(p=.005)$ in RMTs.

In the validation study, we performed grid- and gyrus-based protocols to map the cortical representations of FDI (intensity of 105\% RMT of FDI), EDC (intensity of 105\% RMT of EDC) and FCR (intensity of 105\% RMT of FCR) in Session 1; cf. Figure 3 and Figure S1 in the Supplementary Material. The differences in $C_{0} G_{x, y, z}$ between grid- and gyrus-based mapping was $\pm 5 \mathrm{~mm}$ for most subjects in all three intensities, without systematic effect for the $\operatorname{Co}_{x, y, z}$ location. The amplitude differences between the two protocols were usually in the region of $\pm 100 \mu \mathrm{V}$, while the latency differences were typically in the range of $\pm 1.5 \mathrm{~ms}$ for all the subjects. The size of the active area showed larger differences between grid-and gyrus-based mapping, with the analysis of the slope indicating that the differences in the size of the active area between the two methods increased when the active area increased (test for slopes, FDI t(18) $=2.91, p=.010, \operatorname{EDC~t}(20)=4.00, p=.0008$, and FCR $\mathrm{t}(20)=3.89, p=.001)$; see Figure $\mathrm{S} 1$ in the Supplementary Material for more details. 

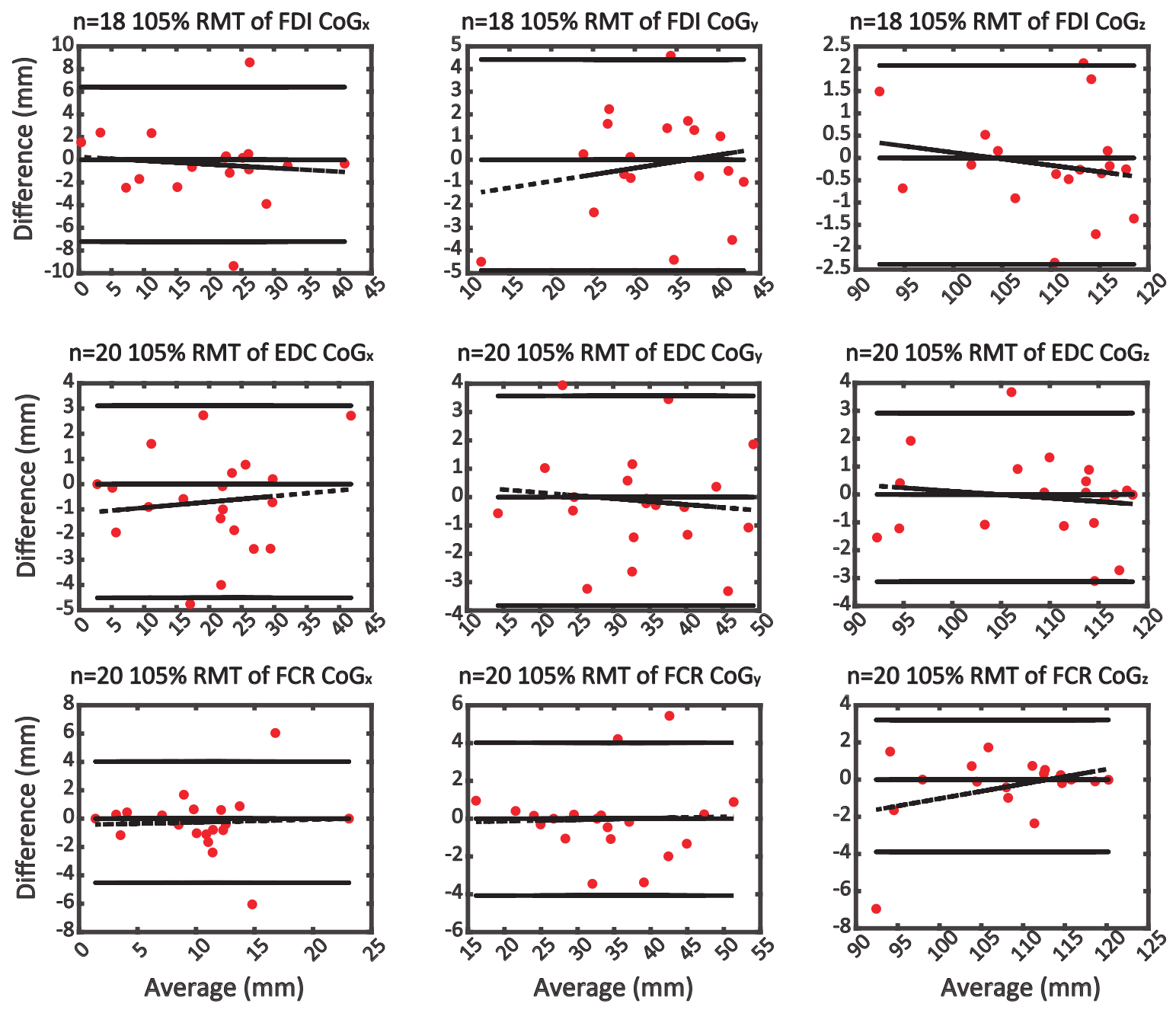

Figure 3. Comparisons of the grid- and the gyrus-based protocols. The panels show the Bland-Altman plots for the parameters of $C_{o} G_{x, y, z}$ for the three intensities. The coloured points represent the difference between the results of the gyrus- and grid-based protocol.

\section{Between session reliability}

Table 1 provides an overview of the ICCs of the gyrus-based mapping between Session 1 and 2 of the MEP amplitude and latency, as well as of the $\operatorname{Co}_{x, y, z}$ and the size of the active area for the stimulation intensity based on EDC-RMT. ICC values for the mappings based on the other muscles RMT are shown in Table S2 and Table S3 in the Supplementary Material.

Table 1. Intraclass correlation coefficients (ICCS) indicating the reliability of the estimated MEP amplitude and latency and $\operatorname{CoG}_{x, y, z}$ and the size of the active area at stimulation intensity of $105 \%$ RMT of EDC.

\begin{tabular}{|c|c|c|c|c|c|c|c|c|}
\hline & FDI & ADM & APB & FPB & EDC & FDS & ECR & FCR \\
\hline Amplitude & 0.93 & 0.83 & 0.78 & 0.87 & 0.84 & 0.84 & 0.98 & 0.77 \\
\hline Latency & 0.97 & 0.91 & 0.65 & 0.59 & 0.80 & 0.82 & 0.92 & 0.84 \\
\hline $\operatorname{CoG}_{x}$ & 0.85 & 0.84 & 0.86 & 0.86 & 0.88 & 0.86 & 0.87 & 0.85 \\
\hline $\operatorname{CoG}_{y}$ & 0.70 & 0.79 & 0.78 & 0.82 & 0.76 & 0.76 & 0.74 & 0.74 \\
\hline $\operatorname{CoG}_{z}$ & 0.87 & 0.88 & 0.88 & 0.91 & 0.89 & 0.92 & 0.87 & 0.88 \\
\hline Active area & 0.63 & 0.64 & 0.65 & 0.75 & 0.43 & 0.58 & 0.77 & 0.48 \\
\hline
\end{tabular}


bioRxiv preprint doi: https://doi.org/10.1101/2021.07.29.454279; this version posted July 30, 2021. The copyright holder for this preprint (which was not certified by peer review) is the author/funder, who has granted bioRxiv a license to display the preprint in perpetuity. It is made available under aCC-BY 4.0 International license.

In Figure 4, we illustrate the cortical representations of the eight muscles in Figure 4 based on EDC-

FDI

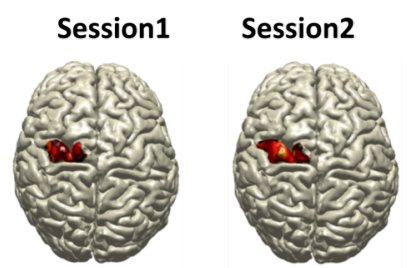

FPB

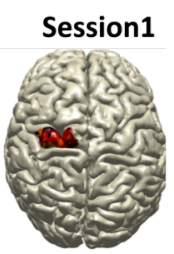

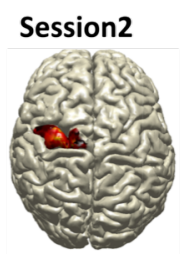

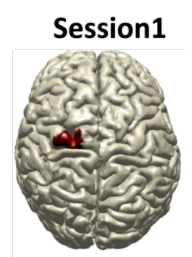

ECR

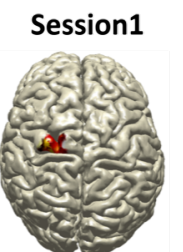

ADM

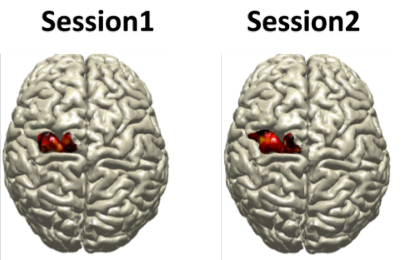

EDC
APB
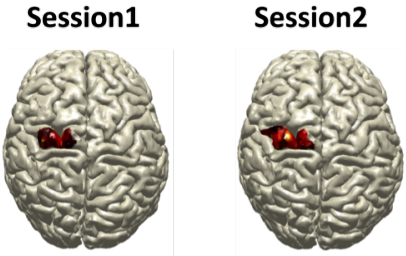

FDS
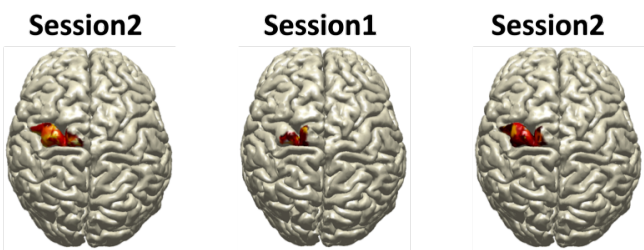

FCR
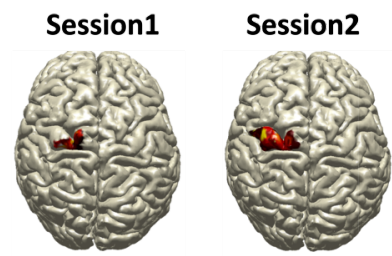

Figure 4. Cortical representation of the eight muscles in a representative subject for the intensity of $105 \%$ RMT of EDC. The cortical representation from Session 1 is depicted on the left panel, whereas the cortical representation from Session 2 is depicted on the right panel. MEP amplitudes colour-code the active area.

The reliability of gyrus-based mapping at different stimulation intensities is summarised in Table 2.

Table 2. The number of muscles in the four levels of ICCs cross the three intensities. ICC was interpreted as follows: $<0.5=$ poor, $0.5-0.64=$ moderate, $0.65-0.79=$ good and $>=0.8$ was excellent.

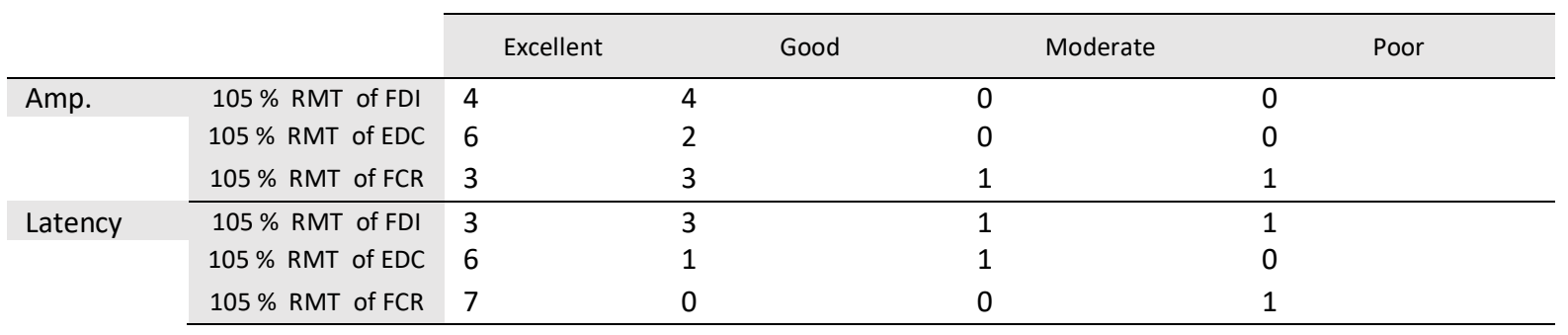




\begin{tabular}{|c|c|c|c|c|c|}
\hline \multirow[t]{3}{*}{$\operatorname{CoG}_{x}$} & $105 \%$ RMT of FDI & 8 & 0 & 0 & 0 \\
\hline & $105 \%$ RMT of EDC & 8 & 0 & 0 & 0 \\
\hline & $105 \%$ RMT of FCR & 8 & 0 & 0 & 0 \\
\hline \multirow[t]{3}{*}{$\mathrm{CoG}_{y}$} & $105 \%$ RMT of FDI & 8 & 0 & 0 & 0 \\
\hline & $105 \%$ RMT of EDC & 1 & 7 & 0 & 0 \\
\hline & $105 \%$ RMT of FCR & 0 & 8 & 0 & 0 \\
\hline \multirow[t]{3}{*}{$\mathrm{CoG}_{z}$} & $105 \%$ RMT of FDI & 8 & 0 & 0 & 0 \\
\hline & $105 \%$ RMT of EDC & 8 & 0 & 0 & 0 \\
\hline & $105 \%$ RMT of FCR & 8 & 0 & 0 & 0 \\
\hline \multirow[t]{3}{*}{ Active area } & $105 \%$ RMT of FDI & 0 & 1 & 6 & 1 \\
\hline & $105 \%$ RMT of EDC & 0 & 3 & 3 & 2 \\
\hline & $105 \%$ RMT of FCR & 0 & 3 & 3 & 2 \\
\hline
\end{tabular}

214 We did not find any significant effects of Intensity or Session (nor of their interaction) on $\operatorname{CoG}_{x, y, z}$ (see 215 also Table S4 in the Supplementary Material). However, the size of the active area for each muscle 216 differed significantly across intensities, without significant effects of Session or Intensity $\times$ Session in217 teraction. The post-hoc pairwise comparisons revealed that the active area tested in the intensity of $218105 \%$ RMT of FCR was markedly larger than in the other intensities; cf. Table 3. For several muscles, 219 the amplitude and latency varied significantly across the intensities (see Table S4 in the Supplementary 220 Material for details).

Table 3. The statistical results of the size of the active area: the average values, the outcomes of the repeated ANOVA (for the factors of Intensity, Session and IntensityxSession) and the pairwise comparisons in the intensity 105\% RMT of FDI 105\% RMT of EDC, 105\% RMT of FDI 105\% RMT of FCR and 105\% RMT of EDC 105\% RMT of FCR.

\begin{tabular}{|c|c|c|c|c|c|c|c|c|c|c|c|c|}
\hline & \multicolumn{3}{|c|}{ Average of active area $\left(\mathrm{mm}^{3}\right)$} & \multirow{2}{*}{$\begin{array}{l}\text { Intensity } \\
F(2,34)\end{array}$} & \multirow[b]{2}{*}{$\begin{array}{c}p- \\
\text { value }\end{array}$} & \multirow{2}{*}{$\begin{array}{l}\text { Session } \\
F(1,17)\end{array}$} & \multirow[b]{2}{*}{$\begin{array}{c}p- \\
\text { value }\end{array}$} & \multirow{2}{*}{$\begin{array}{l}\text { Intensity } \\
\times \text { Session } \\
F(2,34)\end{array}$} & \multirow[b]{2}{*}{$\begin{array}{c}p- \\
\text { value }\end{array}$} & \multicolumn{3}{|c|}{$\begin{array}{l}\text { p-value of pairwise } \\
\text { comparisons }\end{array}$} \\
\hline & $\begin{array}{c}105 \% \text { RMT } \\
\text { of FDI }\end{array}$ & $\begin{array}{l}105 \% \mathrm{RMT} \\
\text { of EDC }\end{array}$ & $\begin{array}{c}105 \% \mathrm{RMT} \\
\text { of } \mathrm{FCR}\end{array}$ & & & & & & & $\begin{array}{l}105 \% \\
\text { RMT } \\
\text { FDI } \\
\text { EDC }\end{array}$ & $\begin{array}{c}105 \% \\
\text { RMT } \\
\text { FDI } \\
\text { FCR }\end{array}$ & $\begin{array}{c}105 \% \\
\text { RMT } \\
\text { EDC } \\
\text { FCR }\end{array}$ \\
\hline FDI & $619.61 \pm 65.32$ & $711.47 \pm 62.73$ & $966.43 \pm 77.85$ & 8.798 & 0.0008 & 0.181 & 0.676 & 0.385 & 0.684 & 0.750 & 0.006 & 0.023 \\
\hline ADM & $439.54 \pm 80.66$ & $495.22 \pm 73.36$ & $688.80 \pm 86.01$ & 4.735 & 0.015 & 0.781 & 0.389 & 0.057 & 0.945 & 1.000 & 0.061 & 0.131 \\
\hline APB & $576.17 \pm 91.58$ & $619.43 \pm 68.58$ & $885.56 \pm 66.29$ & 6.515 & 0.004 & 0.011 & 0.918 & 0.748 & 0.481 & 1.000 & 0.024 & 0.027 \\
\hline FPB & $580.83 \pm 98.33$ & $661.24 \pm 79.00$ & $878.70 \pm 69.46$ & 6.177 & 0.005 & 0.299 & 0.592 & 0.287 & 0.752 & 0.859 & 0.859 & 0.062 \\
\hline EDC & $652.80 \pm 94.43$ & $694.80 \pm 59.15$ & $933.34 \pm 89.09$ & 6.391 & 0.004 & 0.861 & 0.366 & 0.039 & 0.962 & 1.000 & 0.029 & 0.024 \\
\hline FDS & $565.54 \pm 83.40$ & $583.56 \pm 70.26$ & $834.56 \pm 76.11$ & 5.642 & 0.008 & 4.145 & 0.058 & 0.513 & 0.603 & 1.000 & 0.026 & 0.045 \\
\hline ECR & $572.26 \pm 95.14$ & $567.46 \pm 73.18$ & $871.06 \pm 82.29$ & 8.216 & 0.001 & 0.365 & 0.554 & 0.023 & 0.978 & 1.000 & 0.009 & 0.005 \\
\hline FCR & $531.05 \pm 89.14$ & $530.23 \pm 76.39$ & $791.67 \pm 78.86$ & 5.201 & 0.011 & 1.745 & 0.204 & 0.518 & 0.601 & 1.000 & 0.028 & 0.062 \\
\hline
\end{tabular}

\section{Discussion}

222 We designed a protocol to investigate the cortical representation of multiple muscles using navigated single-pulse TMS. Conventional grid-based mapping and our gyrus-based mapping were performed using three stimulation intensities in two consecutive sessions. We determined RMTs of eight muscles as well as MEP amplitudes and latencies, the centre-of-gravity and the size of the corresponding active areas. We found that our new protocol is as valid and as reliable as the commonly applied grid-based approach but appears much more feasible. Our protocol reduces assessment times and simplifies ex- 
perimental procedures. In our experiment, it took only about 10 minutes to map eight muscles simultaneously ( 5 second intervals $\times 120$ points $/ 60$ ), while for the conventional grid-based approach with serial muscle assessment, at least 80 minutes would be spent. Experimenting over such a long period is prone to navigational errors and aggravates participants' fatigue. Moreover, by construction separate measurements will not provide reliable insight about overlapping cortical muscle representations.

One of the problems in designing multiple muscles mapping is that the RMT of a single muscle is considered a reference when setting the stimulation intensity. We found that the RMTs of the hand and forearm muscles considered are indeed marginally different (EDC-FCR and FDI-FCR). The average RMTs indicated that the difference between RMTs is small (not more than $3.1 \%$ of stimulator output). Intensities of $105 \%, 110 \%$ to $120 \%$ [27] RMT have been widely used in motor mapping [8, 27, 28], suggesting that the here-observed difference is acceptable if not negligible. Hence, forearm and hand muscles might be pooled in a group of muscles with "similar RMTs" and may indeed be evaluated at the same intensity.

The validity testing clearly revealed that our gyrus-based mapping agrees with the grid-based standard, consistent with a previous study on pseudorandom stimulus positioning [20,29]. The variation of $\operatorname{Co}_{x, y, z}$ values between the two protocols was restricted to a range of about $-4 \mathrm{~mm}$ to $4 \mathrm{~mm}$. The differences in size of the active area between protocols are more considerable, and the betweensubjects variability is clearly noteworthy. One explanation for this is that any outlier stimulation site may strongly affect the estimate of the active area. Moreover, the individual MRIs profoundly differed in size. While the first calls for more statistical evaluation and (spatial) outlier detection, the latter will soon be addressed by projecting the individual MRI to, e.g., the MNI template [13].

The reliability of amplitude and latency were excellent or good for all the muscles and all the parameters analysed in APB and ECR achieved excellent to good ICCS, indicating the required reliability of our approach. The ICC values of the size of the active area in FDI, ADM, EDC and FCR were moderate to poor. This may be related to the factors. First, in some participants, the RMTs of these muscles were higher than the intensities we set. That is, the intensity of stimulation was too low to activate the corresponding cortical areas. Second, one must realise that even a figure-of-eight coil comes with a widespread focus. Hence, TMS activates not isolated but also nearby areas. Yet, the estimated centresof-gravity appeared very consistent and should be considered reliable parameters in motor mapping, in line with previous studies [20,21].

A gyrus-based protocol with nTMS for multiple muscles allows for not just assessing multiple muscles in parallel, especially when stepping away from mere "active point" drawing [30] to estimating muscle- 
specific active areas in detail. Isolated muscles are not mapped to isolated cortical areas, but one determines the cortical representation of a set of muscles. Results show that these cortical representation manifest overlaps that diverges across the muscles [31]; cf. Figure 4. This comes particularly to the fore when analysing cortical surfaces in 3D. There are several methods to determine mapping areas using nTMS $[19,32]$ that stand out for their computational ease. However, by ignoring the 3D representation of surface folds, one runs the risk of missing important information about active areas in the gyri vis-à-vis the sulci. We used a 3D-alternative (as explained in the Supplementary Material) that overcomes these potential shortcomings. This also provides a welcome visualisation of the active area on the cortex (for single or multiples muscles), which might serve as a convenient tool. In future research, we will exploit its capacity to unravel the nature of the overlap region of active areas of multiple muscles.

We selected three intensities when eliciting MEPs, namely 105\% RMT of FDI as a representative of the hand muscle, EDC as extensor and FCR for flexor muscles. Despite the small variation in RMTs of all muscles, one may still consider this sub-optimal. Image-based RMT prediction may be an excellent way to address this issue. This should involve modelling the electric field distributions of TMS, which may also serve to quantify overlapping cortical representation with even higher precision than currently possible.

\section{Conclusion}

We designed a time-efficient protocol for identifying the cortical representation of multiple muscles in parallel. Our procedure takes only about ten minutes per subject when including eight muscles. The results of the active area's centre-of-gravity at three distinct stimulation intensities can be considered very reliable. All the here-considered outcome parameters confirm the validity of the procedure when compared to a conventional, grid-based approach with muscle-specific resting motor threshold-scaled stimulation intensities.

\section{Acknowledgements}

The authors are thankful to Moira van Leeuwen, who provided assistance during the measurements. Sjoerd Bruijn was funded by a grant from the Netherlands Organization for Scientific Research (016.Vidi.178.014), https://www.nwo.nl/en/. 


\section{References}

[1] Barker AT, Jalinous R, Freeston IL. Non-invasive magnetic stimulation of human motor cortex. The Lancet 1985;325(8437):1106-7.

[2] Schambra H, Sawaki L, Cohen L. Modulation of excitability of human motor cortex (M1) by $1 \mathrm{~Hz}$ transcranial magnetic stimulation of the contralateral M1. Clinical Neurophysiology 2003;114(1):130-3.

[3] Rossini PM, Burke D, Chen R, Cohen L, Daskalakis Z, Di lorio R, et al. Non-invasive electrical and magnetic stimulation of the brain, spinal cord, roots and peripheral nerves: basic principles and procedures for routine clinical and research application. An updated report from an IFCN Committee. Clinical Neurophysiology 2015;126(6):1071-107.

[4] Rossini PM, Barker A, Berardelli A, Caramia M, Caruso G, Cracco R, et al. Non-invasive electrical and magnetic stimulation of the brain, spinal cord and roots: basic principles and procedures for routine clinical application. Report of an IFCN committee. Electroencephalography and clinical neurophysiology 1994;91(2):79-92.

[5] Siebner $\mathrm{H}$, Rothwell J. Transcranial magnetic stimulation: new insights into representational cortical plasticity. Experimental brain research 2003;148(1):1-16.

[6] Krieg SM, Shiban E, Buchmann N, Gempt J, Foerschler A, Meyer B, et al. Utility of presurgical navigated transcranial magnetic brain stimulation for the resection of tumors in eloquent motor areas. Journal of neurosurgery 2012;116(5):994-1001.

[7] Mark V, Taub E, Morris D. Neuroplasticity and constraint-induced movement therapy. Europa medicophysica $2006 ; 42(3): 269$.

[8] Tarapore PE, Tate MC, Findlay AM, Honma SM, Mizuiri D, Berger MS, et al. Preoperative multimodal motor mapping: a comparison of magnetoencephalography imaging, navigated transcranial magnetic stimulation, and direct cortical stimulation. Journal of neurosurgery 2012;117(2):354-62.

[9] Forster M-T, Hattingen E, Senft C, Gasser T, Seifert V, Szelényi A. Navigated transcranial magnetic stimulation and functional magnetic resonance imaging: advanced adjuncts in preoperative planning for central region tumors. Neurosurgery 2011;68(5):1317-25.

[10] Melgari J-M, Pasqualetti P, Pauri F, Rossini PM. Muscles in "concert": study of primary motor cortex upper limb functional topography. PloS one 2008;3(8):e3069.

[11] Wassermann EM, McShane LM, Hallett M, Cohen LG. Noninvasive mapping of muscle representations in human motor cortex. Electroencephalography and Clinical Neurophysiology/Evoked Potentials Section 1992;85(1):1-8.

[12] Gugino LD, Romero JR, Aglio L, Titone D, Ramirez M, Pascual-Leone A, et al. Transcranial magnetic stimulation coregistered with MRI: a comparison of a guided versus blind stimulation technique and its effect on evoked compound muscle action potentials. Clinical neurophysiology 2001;112(10):1781-92.

[13] Kraus D, Gharabaghi A. Projecting navigated TMS sites on the gyral anatomy decreases inter-subject variability of cortical motor maps. Brain stimulation 2015;8(4):831-7.

[14] Vink JJ, Petrov PI, Mandija S, Dijkhuizen RM, Neggers SF. Outcome of TMS-based motor mapping depends on TMS current direction. bioRxiv 2018:371997.

[15] Julkunen P, Säisänen L, Danner N, Niskanen E, Hukkanen T, Mervaala E, et al. Comparison of navigated and non-navigated transcranial magnetic stimulation for motor cortex mapping, motor threshold and motor evoked potentials. Neuroimage 2009;44(3):790-5.

[16] Krieg SM, Lioumis P, Mäkelä JP, Wilenius J, Karhu J, Hannula H, et al. Protocol for motor and language mapping by navigated TMS in patients and healthy volunteers; workshop report. Acta neurochirurgica 2017;159(7):1187-95.

[17] Kleim JA, Kleim ED, Cramer SC. Systematic assessment of training-induced changes in corticospinal output to hand using frameless stereotaxic transcranial magnetic stimulation. Nature Protocols 2007;2(7):1675. 
bioRxiv preprint doi: https://doi.org/10.1101/2021.07.29.454279; this version posted July 30,2021 . The copyright holder for this preprint (which was not certified by peer review) is the author/funder, who has granted bioRxiv a license to display the preprint in perpetuity. It is made available under aCC-BY 4.0 International license.

[18] Littmann AE, McHenry CL, Shields RK. Variability of motor cortical excitability using a novel mapping procedure. Journal of neuroscience methods 2013;214(2):137-43.

[19] Van De Ruit M, Perenboom MJ, Grey MJ. TMS brain mapping in less than two minutes. Brain stimulation 2015;8(2):231-9.

[20] Cavaleri R, Schabrun SM, Chipchase LS. The reliability and validity of rapid transcranial magnetic stimulation mapping. Brain stimulation 2018.

[21] Weiss C, Nettekoven C, Rehme AK, Neuschmelting V, Eisenbeis A, Goldbrunner R, et al. Mapping the hand, foot and face representations in the primary motor cortex-retest reliability of neuronavigated TMS versus functional MRI. Neuroimage 2013;66:531-42.

[22] Rossi S, Hallett M, Rossini PM, Pascual-Leone A. Screening questionnaire before TMS: an update. 2011.

[23] Oldfield RC. The assessment and analysis of handedness: the Edinburgh inventory. Neuropsychologia 1971;9(1):97-113.

[24] Groppa S, Oliviero A, Eisen A, Quartarone A, Cohen L, Mall V, et al. A practical guide to diagnostic transcranial magnetic stimulation: report of an IFCN committee. Clinical Neurophysiology 2012;123(5):858-82.

[25] Malcolm M, Triggs W, Light K, Shechtman O, Khandekar G, Rothi LG. Reliability of motor cortex transcranial magnetic stimulation in four muscle representations. Clinical neurophysiology 2006;117(5):1037-46.

[26] Opitz A, Zafar N, Bockermann V, Rohde V, Paulus W. Validating computationally predicted TMS stimulation areas using direct electrical stimulation in patients with brain tumors near precentral regions. Neurolmage: Clinical 2014;4:500-7.

[27] Akiyama T, Ohira T, Kawase T, Kato T. TMS orientation for NIRS-functional motor mapping. Brain topography 2006;19(1-2):1-9.

[28] Bohning D, He L, George M, Epstein C. Deconvolution of transcranial magnetic stimulation (TMS) maps. Journal of neural transmission 2001;108(1):35-52.

[29] Jonker ZD, van der Vliet R, Hauwert CM, Gaiser C, Tulen JH, van der Geest JN, et al. TMS motor mapping: Comparing the absolute reliability of digital reconstruction methods to the golden standard. Brain stimulation 2019;12(2):309-13.

[30] Seynaeve L, Haeck T, Gramer M, Maes F, De Vleeschouwer S, Van Paesschen W. Optimised preoperative motor cortex mapping in brain tumors using advanced processing of transcranial magnetic stimulation data. Neurolmage: Clinical 2019;21:101657.

[31] Mathew J, Kübler A, Bauer R, Gharabaghi A. Probing corticospinal recruitment patterns and functional synergies with transcranial magnetic stimulation. Frontiers in cellular neuroscience 2016;10:175.

[32] Julkunen P. Methods for estimating cortical motor representation size and location in navigated transcranial magnetic stimulation. Journal of neuroscience methods 2014;232:125-33. 